\title{
Co-culture with endometrial stromal cells enhances the differentiation of human embryonic stem cells into endometrium-like cells
}

\author{
WENZHU YU $^{1 *}$, WENBIN NIU ${ }^{1 *}$, SHUNA WANG $^{1}$, XUEMEI CHEN ${ }^{2}$, \\ BO SUN ${ }^{1}$, FANG WANG $^{1}$ and YINGPU SUN ${ }^{1}$ \\ ${ }^{1}$ Reproductive Medical Center, The First Affiliated Hospital of Zhengzhou University, Zhengzhou, Henan 450052; \\ ${ }^{2}$ Department of Human Anatomy, College of Basic Medical Sciences, \\ Zhengzhou University, Zhengzhou, Henan 450001, P.R. China
}

Received December 23, 2014; Accepted April 8, 2015

DOI: 10.3892/etm.2015.2490

\begin{abstract}
In vitro differentiation of human embryonic stem cells (hESCs) into endometrium-like cells may provide a useful tool for clinical treatment. The aim of the present study was to investigate the differentiation potential of hESCs into endometrium-like cells using three methods, which included induction by feeder cells, co-culture with endometrial stromal cells and induction with embryoid bodies. Following differentiation, the majority of cells positively expressed cytokeratin and epithelial cell adhesion molecule (EPCAM). Factors associated with endometrium cell function, namely the estrogen and progesterone receptors (ER and PR), were also detected. At day 21 following the induction of differentiation, the expression levels of cytokeratin, EPCAM, ER and PR were significantly increased in the co-culture method group, as compared with the other two methods. Furthermore, these cells became decidualized in response to progesterone and prolactin. In addition, the number of cytokeratin-positive or EPCAM-positive cells significantly increased following the induction of differentiation using the co-culture method, as compared with the other two methods. The mRNA expression levels of Wnt members that are associated with endometrial development were subsequently examined, and Wnt5a was found to be significantly upregulated in the differentiated cells induced by feeder cells and co-culture with endometrial stromal cells; however, Wnt4 and Wnt7a expression levels were unaffected. Additionally, the mRNA expression levels of
\end{abstract}

Correspondence to: Professor Yingpu Sun, Reproductive Medical Center, The First Affiliated Hospital of Zhengzhou University, 1 Jianshe East Road, Zhengzhou, Henan 450052, P.R. China E-mail: doctorsunyingpu@163.com

*Contributed equally

Key words: human embryonic stem cells, co-culture, differentiation, endometrium-like cells, Wnt signaling pathway
Wnt5a in the differentiated cells co-cultured with endometrial stromal cells were higher when compared with those induced by feeder cells. In conclusion, the present findings indicated that the co-culture system is the optimal protocol for the induction of hESC differentiation into endometrium-like cells, and Wnt5a signaling may be involved in this process.

\section{Introduction}

Human embryonic stem cells (hESCs) were first isolated and cultured in 1998 (1). A characteristic feature of hESCs is their capacity to self-renew and differentiate into numerous types of cells. Therefore, hESCs may potentially be used in the therapeutic management of major human diseases, such as neurodegeneration, diabetes and cardiovascular diseases (2). In order to improve the application of stem cell-based strategies for the treatment of destructive diseases, research investigating new territories in the context of hESC maintenance and differentiation is undergoing (3). The prerequisites for the clinical application of hESCs are as follows. Firstly, the derivation, maintenance and differentiation of hESCs should be accomplished under strict aseptic culture conditions. Secondly, the protocol for hESC differentiation into a desired functional cell type should be investigated and validated, in order to ensure the differentiated cells are homogeneous and do not form teratomas or cause cancer. Finally, the transplantation of hESCs or their differentiated derivatives should not induce immune responses or rejection (4-6).

Mature endometrium cells have a limited potential to proliferate in vitro; however, endometrium-like cells derived from hESCs are able to overcome this limitation. Thus, endometrium-like cells derived from hESCs can aid the study of signaling pathways critical to endometrial regeneration, and may ultimately lead to the development of stem cell-based therapies. Although conventional methods are insufficient to induce the differentiation of hESCs into endometrium-like cells, a previous study demonstrated successful induction of the formation of endometrium de novo from bone marrow-derived cells (7). However, the mechanisms underlying the induction of hESC differentiation are not fully understood. 
The coordinated regulation of gene expression and the precise interactions between neighboring cells are critical for the specification and proper arrangement of new cell types during tissue differentiation. This process occurs via at least three types of cell-based interactions, namely cell-cell, cell-extracellular matrix and cell-growth factors/signaling molecules, within the tissues (8). Numerous morphogenetic changes are induced by the engagement of extracellular ligands with their respective receptors (9-11). Soluble and insoluble signaling molecules, along with physiochemical factors, form a tissue niche that promotes cell differentiation toward specific lineages (12). To date, various attempts have been made worldwide to define the optimal culture conditions for hESC growth and differentiation, and numerous cytokines and growth factors, such as Wnt proteins, fibroblast growth factor (FGF), heparin, transforming growth factor (TGF)- $\beta$, insulin-like growth factor (IGF)-II, activin A, platelet-derived grow th factor (PDGF) and neurotrophins have been identified (13-15). The Wnt signaling pathway plays an important role in the development of the endometrium (16). Furthermore, Wnt4, Wnt5a and Wnt7a have been demonstrated to participate in the early development of the reproductive system in females $(17,18)$. However, inducing the differentiation of hESCs into a specific cell type, such as endometrium cells, remains a challenge.

In the present study, the differentiation potential of hESCs into endometrium-like cells was compared under different culture conditions. In addition, the expression of Wnt members during differentiation was determined.

\section{Materials and methods}

Reagents. Recombinant human epidermal growth factor (EGF), type I collagenase, TGF- $\alpha$, FGF and PDGF-BB were purchased from Gibco Life Technologies (Grand Island, NY, USA). Recombinant human 17 $\beta$-E2 and medroxyprogesterone acetate were purchased from Sigma-Aldrich (St. Louis, MO, USA). This study was approved by the Ethics Committee of the First Affiliated Hospital of Zhengzhou University (Zhengzhou, China). Written consent was obtained from the subjects who donated the blastocysts for the isolation of hESCs and those whose endometrium tissue was used.

Preparation of human endometrial stromal cells. Human endometrium tissues were obtained from nine women (age, $32.6 \pm 0.8$ years) who had undergone a hysteroscopy. The female subjects had regular menstrual cycles and had not received exogenous hormones in the three months prior to surgery. Full thickness endometrium $(\sim 5 \mathrm{~mm})$ was scraped from the myometrium and washed in phosphate-buffered saline (PBS) containing 1\% penicillin/streptomycin (Gibco Life Technologies). The samples were subsequently cut into small pieces and digested in medium containing $2 \mathrm{mg} / \mathrm{ml}$ type I collagenase (Sigma-Aldrich) for $1-2 \mathrm{~h}$ at $37^{\circ} \mathrm{C}$. The endometrial cells were cultured in medium at $37^{\circ} \mathrm{C}$, under $5 \% \mathrm{CO}_{2}$ in air. The homogeneity of the stromal cells and epithelial cells was evaluated by immunostaining using specific markers for epithelial (cytokeratin) and stromal cells (vimentin), as described previously (19). Following two passages, the purity of the stromal cells was determined to be $\sim 95 \%$.
hESC culture and differentiation. hESCs were isolated from the inner cell mass of in vitro fertilized blastocysts, which were superfluous to in vitro fertilization cycles (20). hESC lines, ZZU-hESCs-2 and ZZU-hESCs-3 (Zhengzhou University), were cultured in knockout Dulbecco's modified Eagle's medium (DMEM; Gibco Life Technologies), containing 20\% knockout serum replacement, $1 \%$ non-essential amino acids, $2 \mathrm{mM}$ L-glutamine, $0.1 \mathrm{mM} \beta$-mercaptoethanol and $8 \mathrm{ng} / \mathrm{ml}$ FGF. The medium was changed daily and the hESCs were passaged using a mechanical method (a syringe tip cut the hESC clusters into uniform-sized cell clumps for seeding on a new feeder layer) every 4-5 days, as previously described (21).

Differentiation of hESCs into endometrium-like cells was performed using three methods, including induction by feeder cells, co-culture with endometrial stromal cells and embryoid body (EB) induction. The feeder cells method included the differentiation of hESCs via the culture of the cells on a feeder layer of mouse embryonic fibroblasts (MEFs) for 4 days. In this strategy, the hESC clone was grown on the feeder cells for 4 days, after which the culture medium (CM) was changed to differentiation medium (DM). After 7 days, the cells were cultured in DM, containing 5\% serum, for one week. From day 14, the cells were cultured in serum-free DM for 7 days, after which the cells were collected for the assessment of differentiation. Differentiation of hESCs was also induced using a co-culture method, which involved culturing hESCs on a feeder layer of MEFs in the lower compartment of a Transwell system (Corning Life Sciences, Beijing, China), while endometrial stromal cells were cultured in the upper compartment. After 4 days, the CM was replaced with DM (10\% serum, $1 \%$ non-essential amino acids, $10 \mathrm{ng} / \mathrm{ml}$ PDGF-BB, $10 \mathrm{ng} / \mathrm{ml}$ EGF, $10 \mathrm{ng} / \mathrm{ml} \mathrm{TGF-} \alpha$ and $10^{-8} \mathrm{~mol} / \mathrm{l}$ $17 \beta-\mathrm{E} 2$ in DMEM/nutrient mixture F12) and cultured for 7 days. An additional differentiation strategy investigated was the initiation of EB formation. In this procedure, the hESC clone was isolated and an EB was formed, which was subsequently cultured in a $0.1 \%$ gelatin-coated dish with DM for 7 days. Following differentiation, the cells were decidualized by culture with DM containing medroxyprogesterone acetate $\left(10^{-6} \mathrm{~mol} / \mathrm{l}\right)$ for 10 days, after which the expression of prolactin (PRL) was detected.

Reverse transcription-quantitative polymerase chain reaction $(R T-q P C R)$. Total RNA was isolated using an RNA mini kit (Qiagen, Valencia, CA, USA). First-strand cDNA was generated using a PrimeScript ${ }^{\mathrm{TM}} \mathrm{RT}$ reagent kit with a gDNA Eraser (Perfect Real Time; Takara Bio, Inc., Otsu, Japan). The PCR reaction was carried out using $2 \mu \mathrm{l}$ cDNA, $0.2 \mu \mathrm{l}$ sense and antisense primers and SYBR Select Master Mix on a 7500 FAST Real Time PCR System (Applied Biosystems Life Technologies, Foster City, CA, USA). The PCR parameters were set at $50^{\circ} \mathrm{C}$ for $2 \mathrm{~min}, 95^{\circ} \mathrm{C}$ for $2 \mathrm{~min}$, and 40 cycles of $95^{\circ} \mathrm{C}$ for $15 \mathrm{sec}$ and $60^{\circ} \mathrm{C}$ for $1 \mathrm{~min}$. GAPDH was used as the internal control. Gene expression levels were determined using the $2^{-\Delta \Delta \mathrm{Ct}}$ method, where $\Delta \mathrm{Ct}=\left(\mathrm{Ct}_{\text {gene }}-\mathrm{Ct}_{\mathrm{GAPDH}}\right)$ and $\mathrm{Ct}$ was the threshold cycle. The specific primer pairs are listed in Table I.

Immunofluorescence (IF). On days 7, 14 and 21, the differentiated cells were washed with 1X PBS and fixed with $4 \%$ 
Table I. Sequences for specific primers.

\begin{tabular}{|c|c|c|c|}
\hline Gene & Primer sequence $\left(5^{\prime}-3^{\prime}\right)$ & $\begin{array}{l}\text { GenBank accession } \\
\text { number }\end{array}$ & $\begin{array}{c}\text { Annealing } \\
\text { temperature }\left({ }^{\circ} \mathrm{C}\right)\end{array}$ \\
\hline Wnt4 & $\begin{array}{l}\text { F: GCTGGGCTCCAAGTACACC } \\
\text { R: GGCTATCCTGACACACATGC }\end{array}$ & NM_030761.4 & 60 \\
\hline Wnt5a & $\begin{array}{l}\text { F: TTACCACTGCAACTATTGCACC } \\
\text { R: CACAATGAACCTTTAGTTTCCA }\end{array}$ & NM_003392.4 & 62 \\
\hline Wnt7a & $\begin{array}{l}\text { F: CCTGGAGGAGAACATGAAGC } \\
\text { R: CAGTAATTGGGTGACTTCTCG }\end{array}$ & NM_004625.3 & 63 \\
\hline CK-18 & $\begin{array}{l}\text { F: GGAAGATGGCGAGGACTTTA } \\
\text { R: AACTTTGGTGTCATTGGTCTC }\end{array}$ & NM_199187.1 & 59 \\
\hline EPCAM & $\begin{array}{l}\text { F: TGCTGTTATTGTGGTTGTGGTG } \\
\text { R: TACTTTGCCATTCTCTTCTTTCTGG }\end{array}$ & NM_002354.2 & 61 \\
\hline ER- $\alpha$ & $\begin{array}{l}\text { F: TGCCAAGGAGACTCGCTA } \\
\text { R: TCAACATTCTCCCTCCTC }\end{array}$ & NM_001122742.1 & 60 \\
\hline PR & $\begin{array}{l}\text { F: ACACAAAACCTGACACCTCC } \\
\text { R: TACAGCATCTGCCCACTGAC }\end{array}$ & NM_001271161.2 & $60 \alpha$ \\
\hline PRL & $\begin{array}{l}\text { F: GGTGGCGACGACTCCTGGAGCCC } \\
\text { R: GACACCAGACCAACTGGTAATG }\end{array}$ & NM_000948.5 & 61 \\
\hline GAPDH & $\begin{array}{l}\text { F: AGAAGGCTGGGGCTCATTTG } \\
\text { R: AGGGGCCATCCACAGTCTTC }\end{array}$ & NM_002046.4 & 59 \\
\hline
\end{tabular}

F, forward; R, reverse; CK, cytokeratin; EPCAM, epithelial cell adhesion molecule; ER, estrogen receptor; PR, progesterone receptor; PRL, prolactin.

paraformaldehyde for $10 \mathrm{~min}$ at room temperature. Following three washes with $1 \mathrm{X}$ PBS, the cells were permeabilized with $0.1 \%$ Triton X-100 in $1 \mathrm{X}$ PBS for $15 \mathrm{~min}$, and blocked with $10 \%$ horse serum (Gibco Life Technologies) for $30 \mathrm{~min}$ at room temperature. Subsequently, the cell samples were incubated with mouse anti-cytokeratin (1:50; cat. no. P2871; Sigma-Aldrich) and rabbit anti-vimentin (1:50; Santa Cruz Biotechnology, Inc., Dallas, TX, USA) antibodies, or mouse anti-epithelial cell adhesion molecule (EPCAM; 1:50; cat. no. 20160; Abcam, Cambridge, MA, USA) and rabbit anti-vimentin (1:50; cat. no. sc-5565; Santa Cruz Biotechnology, Inc.) antibodies overnight at $4^{\circ} \mathrm{C}$. Following three further washes with $1 \mathrm{X}$ PBS, the cells were incubated with an appropriate secondary antibody (iFluor 488 goat anti-mouse $\mathrm{IgG}$, monoclonal, cat. no. AAT-16448; or Cy3 AffiniPure goat anti-mouse IgG, monoclonal, cat. no. A22210; 1:800; Jackson Immunoresearch Laboratories, Inc., West Grove, PA, USA) for $1 \mathrm{~h}$ at room temperature, and the nuclei were stained with 4',6-diamidino-2-phenylindole (1:5,000, Invitrogen, Life Technologies). The sections were mounted with Prolong medium (Life Technologies), and the images were captured using an LSM 700 inverted confocal microscope (Carl Zeiss Shanghai Co. Ltd., Shanghai, China).

Flow cytometry. The differentiated phenotype of endometrium-like cells was determined using flow cytometry (BD FACS Aria Flow Cytometer; BD Biosciences, San Jose, CA, USA). Differentiated cells were trypsinized and stained with fluorescein isothiocyanate-labeled cytokeratin (BD Biosciences) or Alexa Fluor ${ }^{\circledR} 488$ labeled-EPCAM (Abcam), and analyzed by flow cytometry.
Statistical analysis. Statistical analyses were conducted by SPSS software (version 13.0; SPSS Inc., Chicago, IL, USA). A paired, two-tailed Student's t-test and analyses of variance were performed to determine the statistical significance of the differences between conditions. $\mathrm{P}<0.05$ was considered to indicate a statistically significant difference.

\section{Results}

Co-culture with endometrial stromal cells exhibits the highest efficiency for hESC differentiation into endometrium-like cells. Cell-cell interactions play an essential role in the differentiation of hESCs into specific cell types. In the present study, the differentiation potential of hESCs into endometrium-like cells was investigated using three methods, namely induction by feeder cells, co-culture of hESCs with endometrial stromal cells and EB induced-differentiation. The typical morphology of a hESC clone and endometrial stromal cells is shown in Fig. 1A and B, respectively. The morphologies of the differentiated cells at day 21 following the various methods of differentiation induction are shown in Fig. 1C-E.

To evaluate the differentiation potential of hESCs into endometrium-like cells, the protein expression levels of vimentin, EPCAM and cytokeratin were determined using IF staining. Cytokeratin and EPCAM are markers for epithelial cells, while vimentin is a marker for stromal cells. In the co-culture group, $>85 \%$ of the cells expressed epithelial cell markers and $\sim 10 \%$ of the cells were positive for vimentin $(n=100)$, indicating that although the majority of these differentiated cells were epithelial cells, stromal cells were also present (Fig. 2A and B). In the feeder layer group, 

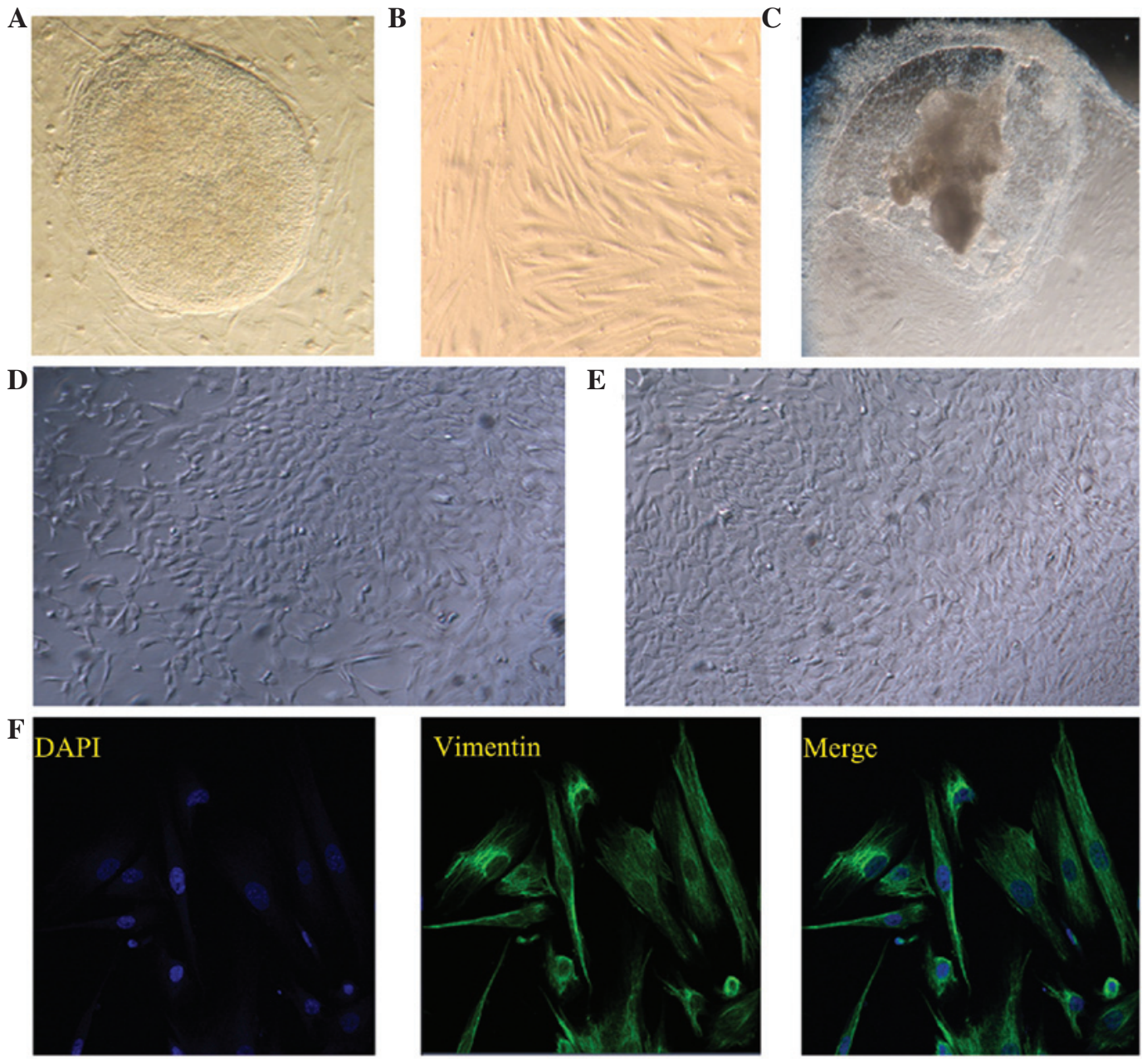

Figure 1. Induction of human embryonic stem cell (hESC) differentiation. Typical morphology of (A) hESC clone and (B) endometrial stromal cells. Images of the differentiated cells on day 21 following induction with (C) embryoid body, (D) mouse embryonic fibroblast feeder cells and (E) co-culture with endometrial stromal cells. Images (A-E) were captured using a Leica inverted microscope (magnification, x200).(F) Immunostaining of vimentin in the endometrial stromal cells, where 4',6-diamidino-2-phenylindole was used to stain the nuclei. Fluorescent images were captured using an LSM 700 inverted confocal microscope (magnification, $\mathrm{x} 400$ ).

almost $70 \%$ of the cells were cytokeratin-positive $(\mathrm{n}=100$ cells); however, in the EB group, only $\sim 40 \%$ of the cells were cytokeratin-positive ( $\mathrm{n}=100$ cells). These observations were further confirmed through flow cytometry (Fig. 3). The numbers of cytokeratin- and EPCAM-positive cells were highest in the co-culture group, when compared with those in the other two groups $(\mathrm{P}<0.05)$.

RT-qPCR analysis was further performed to detect the mRNA expression levels of cytokeratin, EPCAM, estrogen receptor (ER) and progesterone receptor (PR). The expression levels of these genes were significantly increased at day 21 following differentiation induction using the co-culture method, as compared with the other two strategies $(\mathrm{P}<0.05$; Fig. 4). The positive expression of ER and PR indicated that the differentiated cells in the co-culture group may be mediated by estrogen and progesterone hormones. Moreover, the differentiated cells were decidualized by culture with medroxyprogesterone acetate for 10 days, after which the mRNA expression of PRL was detected. The expression of PRL was highest in the co-culture group $(\mathrm{P}<0.05$; Fig. 4), indicating that the differentiated cells in the co-culture group exhibited a superior tendency to decidualization.
Wnt5a is upregulated following hESC differentiation into endometrium-like cells. Wnt4, Wnt5a and Wnt7a have been demonstrated to play important roles in the development of the reproductive system in females. Wnt4 promotes early gender development, Wnt5a promotes uterine gland development and Wnt7a maintains the uterus in normal morphology (22). MEF feeder cells have been found to secrete Wnt proteins (23). Therefore, in order to prevent interference by these proteins secreted by feeder cells or endometrial stromal cells, the mRNA expression levels of Wnt4, Wnt5a and Wnt7a in the EB-induced differentiated cells were examined on days 0,7, 14 and 21. The mRNA expression levels of Wnt4 and Wnt7a were very low, and no statistically significant differences were observed during the process of differentiation $(\mathrm{P}>0.05$; Fig. 5A). However, Wnt5a was significantly upregulated at days 7 and 14 following differentiation induction, with the highest level observed on day $7(\mathrm{P}<0.05)$. This observation indicated that Wnt5a may be associated with the differentiation of hESCs into endometrium-like cells. Accordingly, further investigation into the expression of Wnt5a in the differentiated cells obtained by the various methods was carried out. When compared with the other two methods, the co-culture system 
A

EB

Day 21
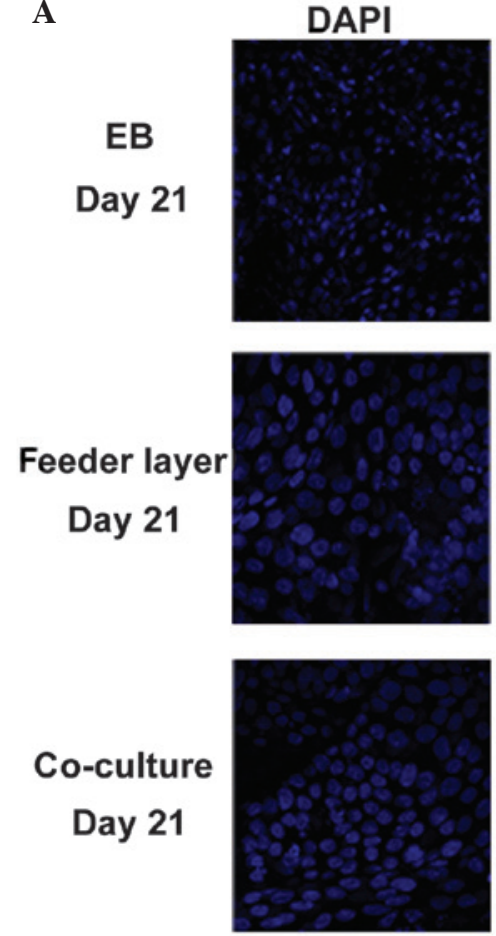

B

EB

Day 21
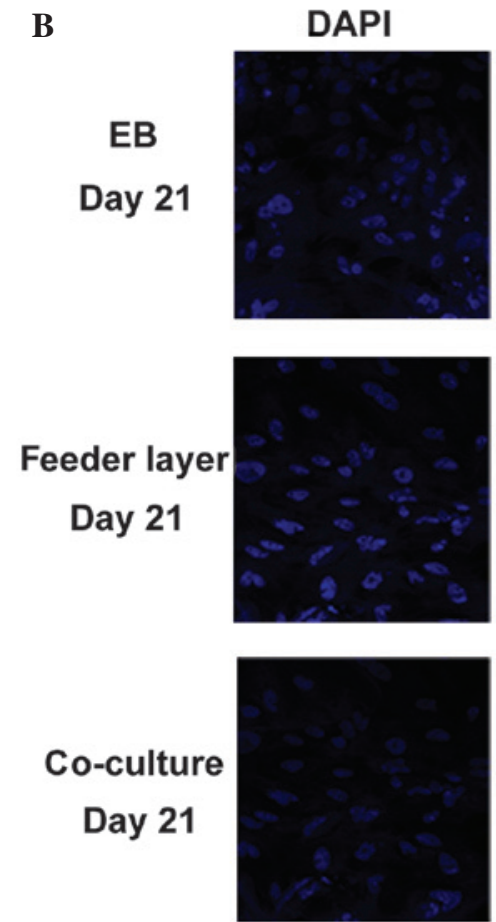
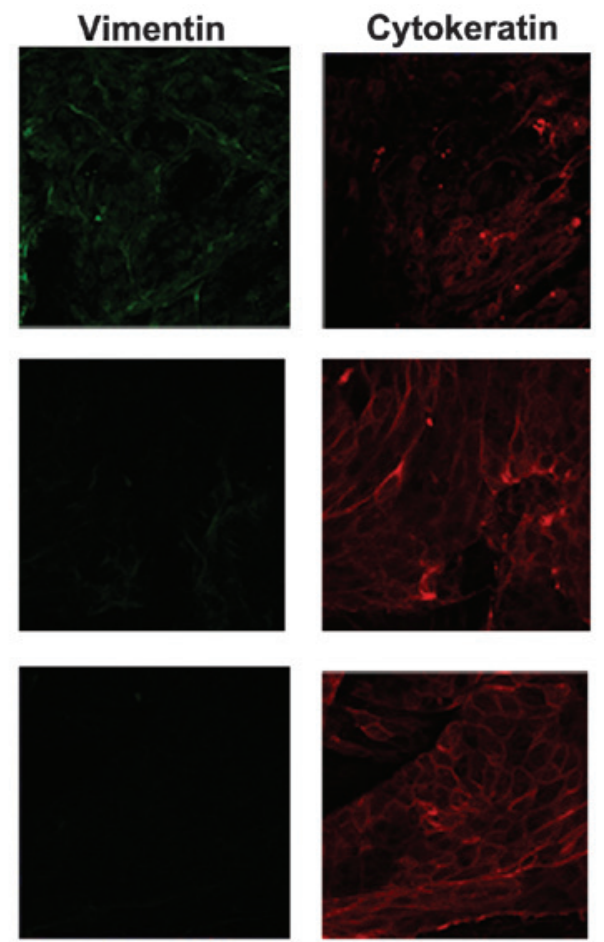

Vimentin
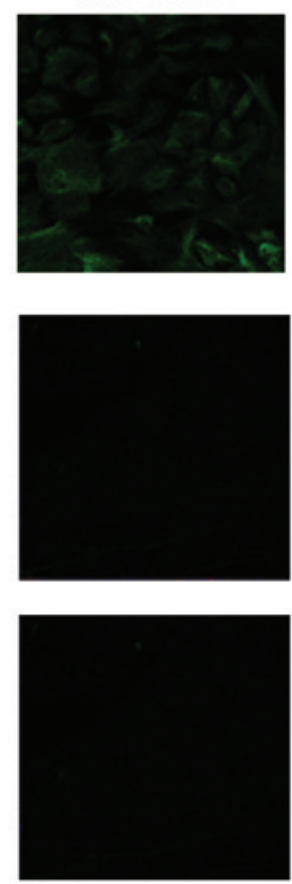

EPCAM
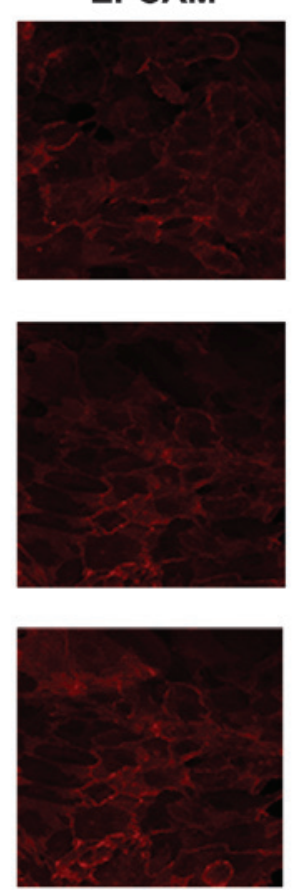

Merge
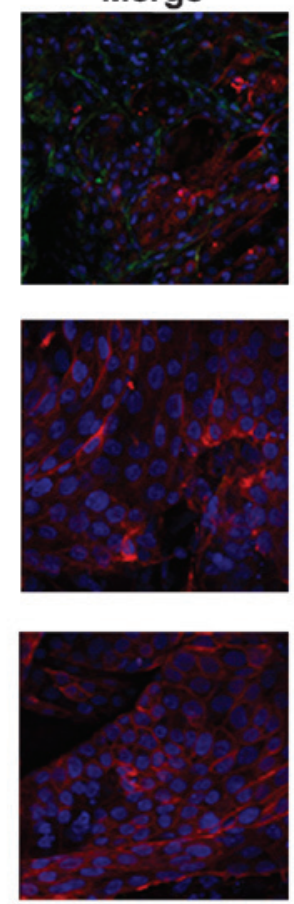

Merge
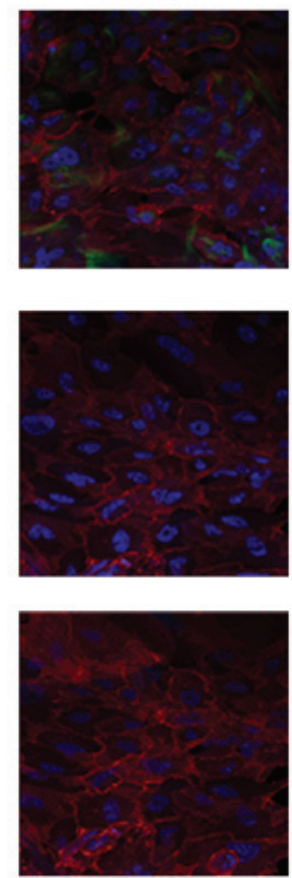

Figure 2. Identification of differentiated cells by immunostaining of vimentin, EPCAM and cytokeratin. Immunostaining of (A) vimentin and cytokeratin and (B) vimentin and EPCAM in the differentiated cells on day 21 following induction by EB, mouse embryonic fibroblast feeder cells or co-culture with endometrial stromal cells. Fluorescent images were captured using an LSM 700 inverted confocal microscope (magnification, x400). EPCAM, epithelial cell adhesion molecule; EB, embryoid body; DAPI, 4',6-diamidino-2-phenylindole.

exhibited a significant upregulation ( $>30$ fold) of Wnt5a expression on day 7 following differentiation induction, followed by a gradual decrease $(\mathrm{P}<0.05$; Fig. $5 \mathrm{~B})$. Furthermore, the mRNA expression levels of Wnt5a were shown to significantly increase between days 7 and 14 following the induction of differentiation in the feeder layer group; however, the expression on day 7 was lower compared with that in the co-culture group ( $\mathrm{P}>0.05$; Fig. 5B).

\section{Discussion}

The self-renewal and differentiation of hESCs requires a large number of endogenous proteins produced by hESCs and exogenous factors in the culture medium (1-3). Routinely, hESCs are cultured on feeder layers of MEFs or on cell-free matrices (laminin or matrigel) supplemented with MEF-conditioned medium (24). An additional approach is to culture hESCs in 
A

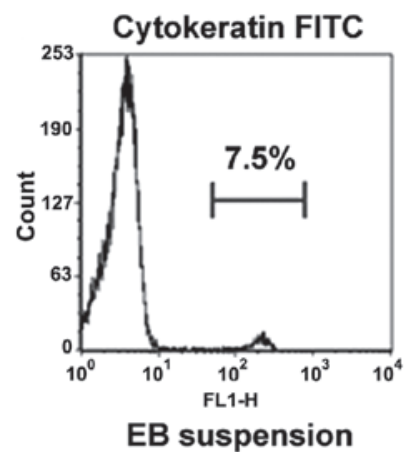

B

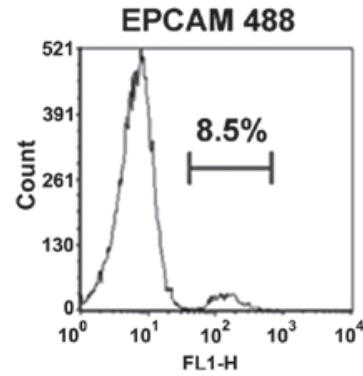

EB suspension
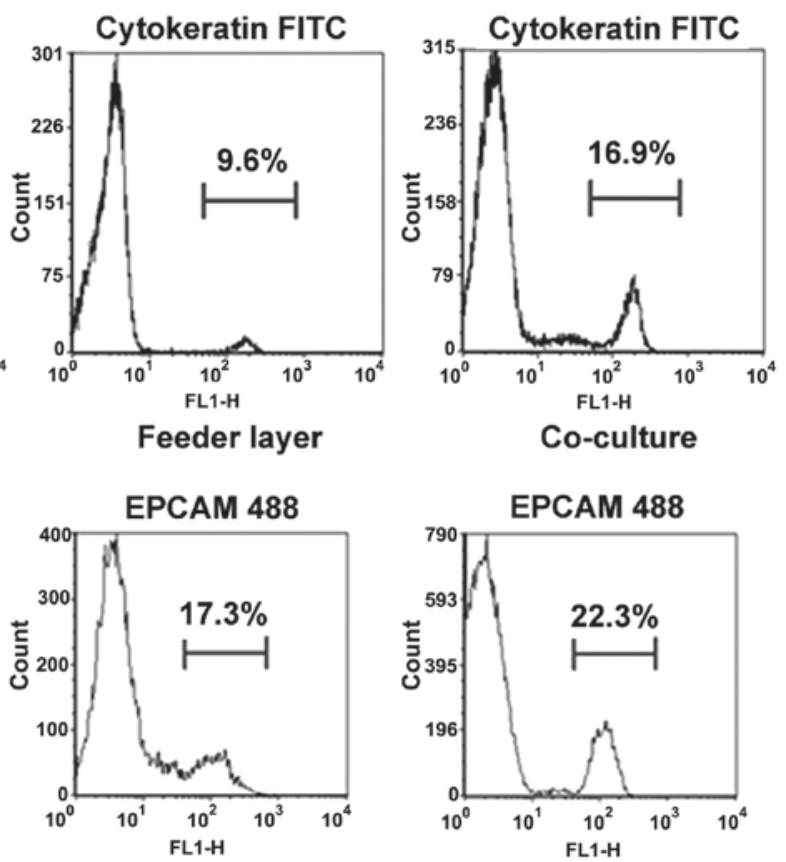

Feeder layer

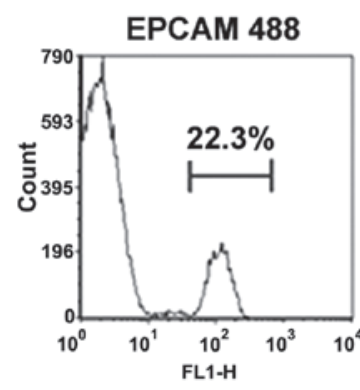

Co-culture

Figure 3. Expression of epithelial cell markers in the differentiated cells, as shown by flow cytometry. Percentage of (A) cytokeratin-positive and (B) EPCAM-positive cells in the differentiated cells on day 21 following induction by EB, mouse embryonic fibroblast feeder cells or co-culture with endometrial stromal cells. EPCAM, epithelial cell adhesion molecule; EB, embryoid body; FITC, fluorescein isothiocyanate.

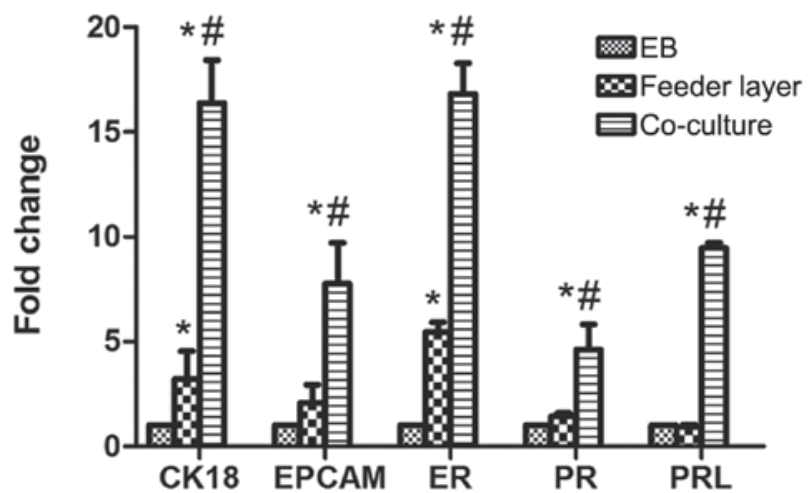

Figure 4. Human embryonic stem cell differentiation into endometrium-like cells. mRNA expression levels of CK18, EPCAM, ER, PR and PRL were analyzed in the differentiated cells on day 21 following induction by EB, mouse embryonic fibroblast feeder cells or co-culture with endometrial stromal cells. Data are presented as the mean \pm standard deviation $(\mathrm{n}=3) .{ }^{*} \mathrm{P}<0.05$, vs. respective EB group; ${ }^{\prime} \mathrm{P}<0.05$, vs. respective feeder layer group. EPCAM, epithelial cell adhesion molecule; ER, estrogen receptor; PR, progesterone receptor; PRL, prolactin; EB, embryonic body; CK, cytokeratin.

suspension conditions that enable hESCs to aggregate and form an EB that is capable of differentiating into three primary germ layers (endoderm, mesoderm and ectoderm), which may be induced to directly differentiate toward the lineage of interest by the induction of growth factors in the culture system (25). However, previous studies have successfully established co-culture systems for hESC differentiation (25). For example, Udayashankar et al (27) successfully generated a cytotrophoblast cell line through co-culture of hESCs with an established endometrial cell culture system. To the best of our knowledge, the present study was the first to compare
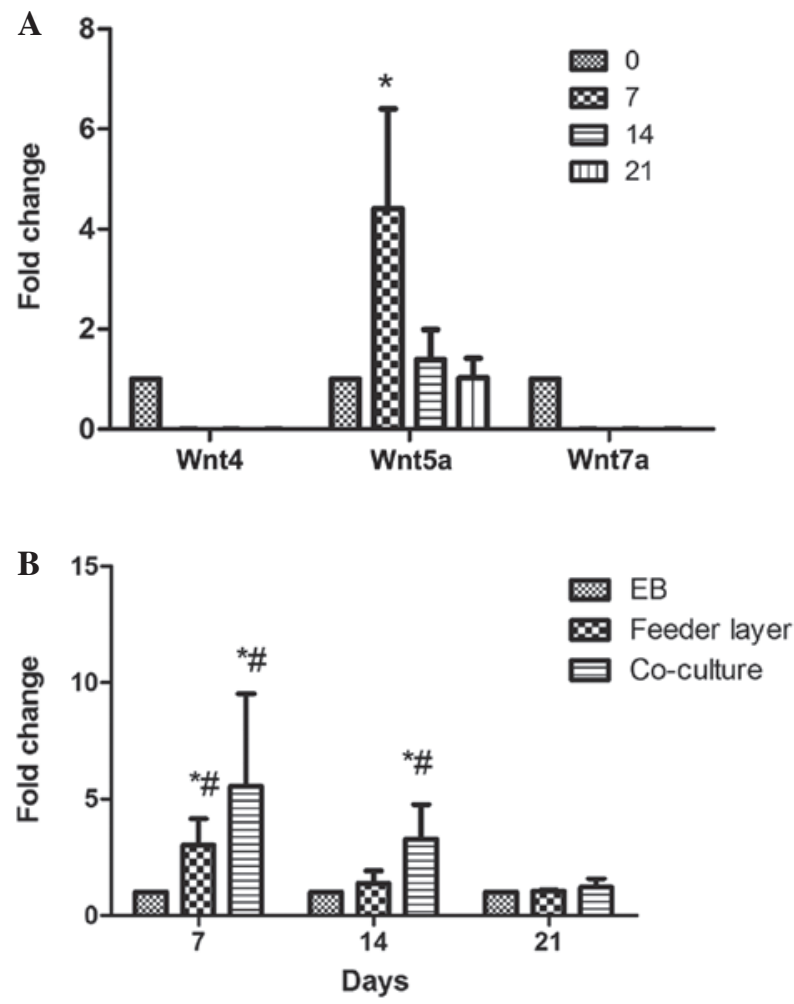

Figure 5. Expression levels of Wnt4, Wnt5a and Wnt7a in the differentiated cells. (A) mRNA expression levels of Wnt4, Wnt5a and Wnt7a in the differentiated cells induced by EB on days $0,7,14$ and 21 . ${ }^{*} \mathrm{P}<0.05$, vs. each of the other groups. (B) Effects of the three methods on the mRNA expression levels of Wnt5a in the differentiated cells. Total RNA from the differentiated cells was isolated on days 7, 14 and 21 and subjected to reverse transcription-quantitative polymerase chain reaction analysis. ${ }^{*} \mathrm{P}<0.05$, vs. each of the other groups at the same time point; ${ }^{\#} \mathrm{P}<0.05$, vs. other time-points in the same group. Data are presented as the mean \pm standard deviation $(n=3)$. EB, embryoid body. 
the differentiation potential of hESCs into endometrium-like cells using three methods, namely EB induced-differentiation, feeder cells and a co-culture system.

In the EB group, the undifferentiated hESCs gradually grew into small cell aggregates and formed a suitably sized EB in suspension conditions. These EB-derived hESCs were able to further differentiate into endometrium-like cells, although the efficacy was lower compared with the other two methods. In addition, MEFs (feeder cells) were demonstrated to promote the differentiation potential of hESCs into endometrium-like cells, which further supported the hypothesis that MEFs contribute to hESC differentiation. Previously, the Transwell co-culture system has been successfully applied to the differentiation of different types of stem cell, such as hESCs, human bone marrow stem cells and human mesenchymal stem cells (27-29). In the present study, the co-culture of hESCs with human endometrial stromal cells was shown to strongly enhance the differentiation potential of hESCs into endometrium-like cells, to a greater extent compared with the other two methods. These observations indicated that the co-culture system may provide superior niches and an improved microenvironment for hESC attachment, expansion and differentiation.

Normal endometrial tissue in the luteal phase is decidualized under the function of progesterone. In addition to changes in cell morphology, the functions of the cells are also altered, such as secretory granules appearing on the cell surface and the secretion of PRL and IGF-binding protein 1. To further investigate the function of the differentiated cells induced by the various methods, the cells were cultured with medroxyprogesterone acetate for 10 days, after which the mRNA expression levels of PRL were detected by RT-qPCR. Although PRL was found to be expressed in all three groups, the expression level was highest in the co-culture group, which is proportional to the efficiency of induced differentiation, indicating that the differentiated cells in the co-culture group exhibited a superior function and were the most sensitive to progesterone.

Yi et al (30) investigated the role of the Wnt signaling pathway in ESCs and showed that transcription factor-3, as a downstream target of the Wnt signaling pathway, may inhibit the promoter activity of Nanog and reduce its expression, subsequently affecting the self-renewal capacity of ESCs and promoting their differentiation. As Wnt signaling is required for embryonic development in invertebrates and vertebrates (31-33), the focus in the present study was on three Wnt members associated with cell proliferation, migration and differentiation. Wnt4, Wnt5a and Wnt7a have also been hypothesized to play important roles in the development of the female reproductive system $(16-18,22)$. In the present study, Wnt5a was revealed to be significantly upregulated in the differentiated cells induced by feeder cells or co-cultured with endometrial stromal cells; however, the expression levels of Wnt4 and Wnt7a were not affected during the differentiation of hESCs into endometrium-like cells induced by the three different methods. Furthermore, the expression level of Wnt5a was consistent with the data regarding the differentiation efficiency in the three groups. Based on the present findings, the co-culture system with endometrial stromal cells was demonstrated to provide the hESCs with a different microenvironment, which lead to an increased activity of the Wnt signaling pathway in the hESCs, through which the efficiency of hESC differentiation into endometrium-like cells was promoted.

In conclusion, the three methods investigated in the present study are able to induce the differentiation of hESCs into endometrium-like cells, with the co-culture system exhibiting the highest efficiency. In addition, Wnt5a expression was found to be significantly upregulated during hESC differentiation into endometrial epithelial-like cells. These observations indicate that the co-culture system is optimal for the induction of hESC differentiation into endometrial cells, and that the Wnt5a signaling may be involved in this process.

\section{References}

1. Thomson JA, Itskovitz-Eldor J, Shapiro SS, et al: Embryonic stem cell lines derived from human blastocysts. Science 282: 1145-1147, 1998.

2. Hu Q and Rosenfeld MG: Epigenetic regulation of human embryonic stem cells. Front Genet 3: 238, 2012.

3. Gill KP, Hewitt AW, Davidson KC, et al: Methods of retinal ganglion cell differentiation from pluripotent stem cells. Transl Vis Sci Technol 3: 7, 2014.

4. Lee JE and Lee DR: Human embryonic stem cells: Derivation, maintenance and cryopreservation. Int J Stem Cells 4: 9-17, 2011.

5. Unger C, Skottman H, Blomberg P, Dilber MS and Hovatta O: Good manufacturing practice and clinical-grade human embryonic stem cell lines. Hum Mol Genet 17: R48-R53, 2008.

6. Mountford JC: Human embryonic stem cells: Origins, characteristics and potential for regenerative therapy. Transfus Med 18: $1-12,2008$

7. Du H and Taylor HS: Contribution of bone marrow-derived stem cells to endometrium and endometriosis. Stem Cells 25: 2082-2086, 2007.

8. Cai L, Ye Z, Zhou BY, Mali P, Zhou C and Cheng L: Promoting human embryonic stem cell renewal or differentiation by modulating Wnt signal and culture conditions. Cell Res 17: 62-72, 2007.

9. Brafman DA, Phung C, Kumar N and Willert K: Regulation of endodermal differentiation of human embryonic stem cells through integrin-ECM interactions. Cell Death Differ 20: 369-381, 2013.

10. Tran NT, Trinh QM, Lee GM and Han YM: Efficient differentiation of human pluripotent stem cells into mesenchymal stem cells by modulating intracellular signaling pathways in a feeder/serum-free system. Stem Cells Dev 21: 1165-1175, 2012.

11. Brafman DA, Chang CW, Fernandez A, Willert K, Varghese S and Chien S: Long-term human pluripotent stem cell self-renewal on synthetic polymer surfaces. Biomaterials 31: 9135-9144, 2010.

12. Jin S, Yao H, Krisanarungson P, Haukas A and Ye K: Porous membrane substrates offer better niches to enhance the Wnt signaling and promote human embryonic stem cell growth and differentiation. Tissue Eng Part A 18: 1419-1430, 2012.

13. Atkinson SP, Lako M and Armstrong L: Potential for pharmacological manipulation of human embryonic stem cells. Br J Pharmacol 169: 269-289, 2013.

14. Dravid G, Ye Z, Hammond H, et al: Defining the role of Wnt/beta-catenin signaling in the survival, proliferation and self-renewal of human embryonic stem cells. Stem Cells 23: 1489-1501, 2005.

15. Pyle AD, Lock LF and Donovan PJ: Neurotrophins mediate human embryonic stem cell survival. Nat Biotechnol 24: 344-350, 2006.

16. Hayashi K and Spencer TE: WNT pathways in the neonatal ovine uterus: potential specification of endometrial gland morphogenesis by SFRP2. Biol Reprod 74: 721-733, 2006.

17. Pellegrino M, Maiorino R and Schonauer S: WNT4 signaling in female gonadal development. Endocr Metab Immune Disord Drug Targets 10: 168-174, 2010.

18. Hayashi K, Yoshioka S, Reardon SN, et al: WNTs in the neonatal mouse uterus: Potential regulation of endometrial gland development. Biol Reprod 84: 308-319, 2011.

19. Malayer JR and Woods VM: Expression of estrogen receptor and maintenance of hormone-responsive phenotype in bovine fetal uterine cells. Domest Anim Endocrinol 15: 141-154, 1998. 
20. Thomson JA, Itskovitz-Eldor J, Shapiro SS, et al: Embryonic stem cell lines derived from human blastocysts. Science 282 : $1145-1147,1998$

21. Chen XM, Kan QC, Wang F, et al: Chromosome dynamic changes in two cultured Chinese human embryonic stem cell lines: Single nucleotide polymorphism, copy number variation and loss of heterozygosity. J Cell Biochem 113: 3520-3527, 2012.

22. Heikkila M, Peltoketo $H$ and Vainio S: Wnts and the female reproductive system. J Exp Zool 290: 616-623, 2001.

23. Xie CQ, Lin G, Luo KL, et al: Newly expressed proteins of mouse embryonic fibroblasts irradiated to be inactive. Biochem Biophys Res Commun 315: 581-588, 2004.

24. Sarkar P, Randall SM, Muddiman DC and Rao BM: Targeted proteomics of the secretory pathway reveals the secretome of mouse embryonic fibroblasts and human embryonic stem cells. Mol Cell Proteomics 11: 1829-1839, 2012.

25. Liu Y, Fox V, Lei Y, Hu B, Joo KI and Wang P: Synthetic niches for differentiation of human embryonic stem cells bypassing embryoid body formation. J Biomed Mater Res B Appl Biomater 102: 1101-1112, 2014.

26. Amirpour N, Nasr-Esfahani MH, Esfandiari E, Razavi S and Karamali F: Comparing three methods of co-culture of retinal pigment epithelium with progenitor cells derived human embryonic stem cells. Int J Prev Med 4: 1243-1250, 2013.
27. Udayashankar R, Baker D, Tuckerman E, Laird S, Li TC and Moore HD: Characterization of invasive trophoblasts generated from human embryonic stem cells. Hum Reprod 26: 398-406, 2011.

28. Murdoch AD, Grady LM, Ablett MP, Katopodi T, Meadows RS and Hardingham TE: Chondrogenic differentiation of human bone marrow stem cells in Transwell cultures: Generation of scaffold-free cartilage. Stem Cells 25: 2786-2796, 2007.

29. Yang Y, Li J, Pan X, et al: Co-culture with mesenchymal stem cells enhances metabolic functions of liver cells in bioartificial liver system. Biotechnol Bioeng 110: 958-968, 2013.

30. Yi F, Pereira L and Merrill BJ: Tcf3 functions as a steady-state limiter of transcriptional programs of mouse embryonic stem cell self-renewal. Stem Cells 26: 1951-1960, 2008.

31. Clevers H: Wnt/beta-catenin signaling in development and disease. Cell 127: 469-480, 2006.

32. van Amerongen $R$ and Nusse R: Towards an integrated view of Wnt signaling in development. Development 136: 3205-3214, 2009.

33. Angers S and Moon RT: Proximal events in Wnt signal transduction. Nat Rev Mol Cell Biol 10: 468-477, 2009. 